\title{
KULEUYEN
}

\section{Early motor intervention in infants with unilateral cerebral palsy: a systematic review and narrative synthesis}

Lisa Mailleux ${ }^{1,2}$, Nathalie De Beukelaer ${ }^{2,3}$, Maria Belén Carbone ${ }^{2}$, Els Ortibus ${ }^{1,4}$

${ }^{1}$ Centre For Developmental Disabilities, Leuven, Belgium; ${ }^{2} \mathrm{KU}$ Leuven, Department of Rehabilitation Sciences, Leuven, Belgium; ${ }^{3}$ University Hospitals Leuven, Clinical Motion Analysis Laboratory, Leuven, Belgium.; ${ }^{4} \mathrm{KU}$ Leuven, Department of Development and Regeneration, Leuven, Belgium.

\section{Background \& Aims}

Due to the higher amount of plasticity of the infant brain, it has been suggested that the benefits from early, activity-based motor interventions are larger compared to the same intervention after two years of life. Hence, studies reporting on the topic of early activity-based motor interventions in infants with unilateral cerebral palsy (uCP) are rising.

The aim of this study was to systematically review the current literature on early motor interventions in infants with uCP.

\section{Methods}

\section{LITERATURE SEARCH:}

We conducted this systematic review following the guidelines of the PRISMA statement (Liberati et al. 2009).

Five electronic databases were screened: PubMed, Embase, Cochrane Central Register of Controlled trials, Cinahl and Web of Science.

After duplicates were removed, search results were assessed for study eligibility by two independent authors (LM and NDB).

Participant and study characteristics were extracted from the selected studies. Critical appraisal was done using the PEDro-scale.

A detailed overview of the selection process is shown in Figure 1

Finally, nine studies were selected including seven intervention studies (i.e. four single blinded randomized controlled trials, one retrospective study, two pre-post designs), one participatory design and one crosssectional survey. Only the results of the intervention studies are presented of which two are from the same study sample (Chamudot et al. 2018).

Figure 1: Flow chart of the selection process

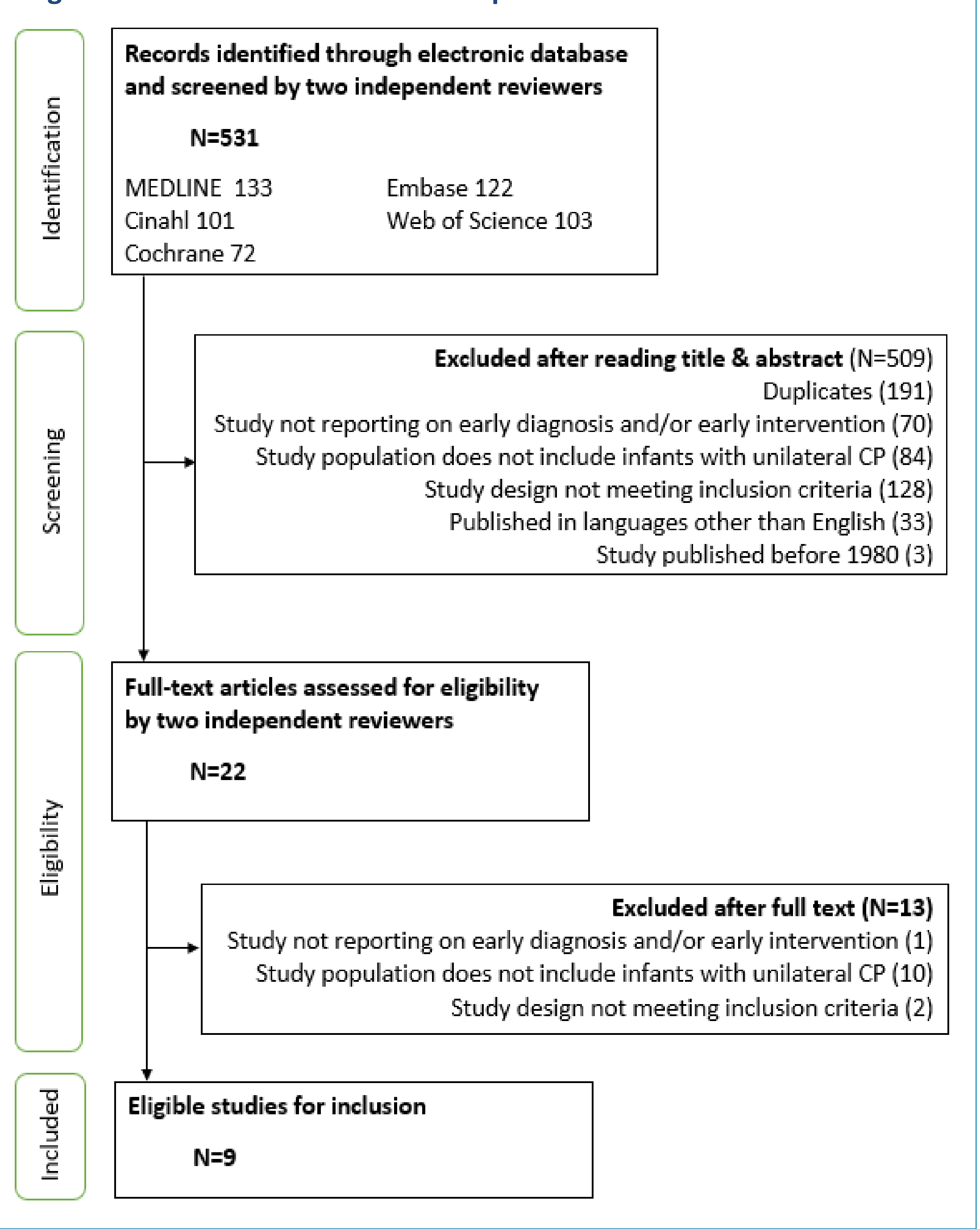

\section{Results}

EARLY MOTOR INTERVENTIONS IN UCP

\begin{tabular}{|c|c|c|c|c|c|}
\hline First author & Study design & Intensity & Therapist & Participants & Study results \\
\hline \multicolumn{6}{|c|}{ Baby-CIMT vs. control } \\
\hline $\begin{array}{l}\text { Hwang et al. } \\
2020\end{array}$ & single blinded RCT & $\begin{array}{l}\text { splint } 3 \text { weeks }+ \\
15 \times 2 \text { hour } \\
\text { sessions }\end{array}$ & OT delivered & $\begin{array}{l}\mathrm{N}=24 \\
\text { Age: } 5-34 \mathrm{~m} \\
14 \mathrm{O}^{\top} ; 10 \text { \% } \\
4 \mathrm{~L} ; 20 \mathrm{R}\end{array}$ & $\begin{array}{l}\text { Improved upper limb use } \\
\text { in real life after CIMT } \\
\text { (parental questionnaire; } \\
\text { Cohen's } d>0.90 \text { ) }\end{array}$ \\
\hline $\begin{array}{l}\text { Nordstrand et al. } \\
2015\end{array}$ & $\begin{array}{l}\text { retrospective non- } \\
\text { RCT }\end{array}$ & $\begin{array}{l}30 \text { minutes/day, } \\
7 \text { days, } 12 \text { weeks }\end{array}$ & $\begin{array}{l}\text { Parent delivered } \\
\text { with weekly OT } \\
\text { guidance }\end{array}$ & $\begin{array}{l}\mathrm{N}=72 \\
\text { Age: }<16 \mathrm{~m} \\
38 \mathrm{o}^{\lambda} ; 34 \text { \& } \\
27 \mathrm{~L} ; 45 \mathrm{R}\end{array}$ & $\begin{array}{l}2 y \text {-old who followed CIMT } \\
\text { had more chance to } \\
\text { achieve higher function } \\
\text { (AHA; Cohen's } d=0.97 \text { ) }\end{array}$ \\
\hline \multicolumn{6}{|c|}{ Baby-CIMT vs. baby-massage } \\
\hline $\begin{array}{l}\text { Eliasson et al. } \\
2018\end{array}$ & single blinded RCT & $\begin{array}{l}30 \text { minutes/day, } \\
6 \text { days, } 12 \text { weeks }\end{array}$ & $\begin{array}{l}\text { Parent delivered } \\
\text { with weekly OT } \\
\text { guidance }\end{array}$ & $\begin{array}{l}\mathrm{N}=31 \\
\text { Age: } 3-8 \mathrm{~m} \\
16 \mathrm{o}^{\pi} ; 15 \text { q } \\
15 \mathrm{~L} ; 16 \mathrm{R}\end{array}$ & $\begin{array}{l}\text { Larger bi- and unimanual } \\
\text { improvements after CIMT } \\
\text { (HAl; Cohen's } d=0.57 \text { and } \\
\text { 0.64) }\end{array}$ \\
\hline \multicolumn{6}{|c|}{ Baby-CIMT vs. bimanual therapy } \\
\hline $\begin{array}{l}\text { Chamudot et al. } \\
2018\end{array}$ & single blinded RCT & $\begin{array}{l}\text { 1hour/day; } \\
7 \text { days, } 8 \text { weeks }\end{array}$ & $\begin{array}{l}\text { Parent delivered } \\
\text { with weekly OT } \\
\text { guidance }\end{array}$ & 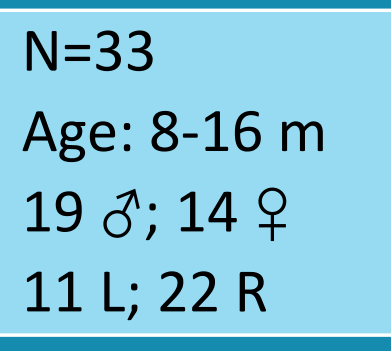 & $\begin{array}{l}\text { Bimanual improvements } \\
\text { independent of therapy } \\
\text { group (mini-AHA; Cohen's } \\
d>2.08 \text { ) }\end{array}$ \\
\hline \multicolumn{6}{|l|}{ eTIPS } \\
\hline $\begin{array}{l}\text { Basu et al. } \\
2018\end{array}$ & pre-post design & $\begin{array}{l}\text { incorporated } \\
\text { daily }\end{array}$ & $\begin{array}{l}\text { Parent delivered } \\
\text { with weekly OT } \\
\text { guidance }\end{array}$ & $\begin{array}{l}\mathrm{N}=13 \\
\text { Age: } 0-6 \mathrm{~m} \\
8 \text { o } ; 5 \text { ㅇ } \\
6 \mathrm{~L} ; 7 \mathrm{R}\end{array}$ & $\begin{array}{l}\text { Feasible and safe to be } \\
\text { delivered by the parents } \\
\text { in the home setting }\end{array}$ \\
\hline \multicolumn{6}{|c|}{ Intensive Task-oriented OT } \\
\hline $\begin{array}{l}\text { Kim et al. } \\
2015\end{array}$ & pre-post design & NR & OT delivered & $\begin{array}{l}\mathrm{N}=36 \\
\text { Age: NR } \\
26 \delta^{\pi} ; 10 \text { ㅇ } \\
\text { NR L; NR R }\end{array}$ & $\begin{array}{l}\text { Improvements after } \\
\text { intervention (HFCS; } p \text { > } \\
0.05 \text { ) }\end{array}$ \\
\hline
\end{tabular}

CIMT: constraint-induced movement therapy; $R C T$, randomized controlled trial; OT, occupational therapist; $N$, number; $m$ months; L, left-sided hemiplegia; $R$, right-sided hemiplegia; HFCS, House Functional Classification Scale.

Three studies reported no adverse events (Basu et al. 2018, Eliasson et al. 2018, Hwang et al. 2020).

Applying CIMT does not appear to delay the function of the restrained hand (Eliasson et al. 2018), nor the infant's gross motor development (Chamudot et al. 2018, Hwang et al. 2020).

\section{PREDICTORS OF TREATMENT RESPONSE}

Baseline function (Eliasson et al. 2018; Chamudot et al. 2018) nor age (Chamudot et al. 2018) were identified as treatment predictors.

The underlying pattern of brain damage was also not a treatment predictor, but only in high-functioning infants. In low-functioning infants, infants with focal infarcts had more chance to develop a very low function (Nordstrand et al. 2015)

Infants with focal infarcts seemed to benefit more from bimanual therapy compared to CIMT, while in white matter lesions treatment response appeared to be independent of the intervention (Chamudot et al. 2018). 